\title{
Gradhiva
}

GRADHIV

Revue d'anthropologie et d'histoire des arts

12 | 2010

La musique n'a pas d'auteur

\section{Araucanie-Bruxelles-Paris : la collection Gustave Verniory au musée du quai Branly}

Témoignages de dix années passées au Chili

Araucania-Brussels-Paris: the Gustave Verniory Collection at the musée du quai Branly. Testament to his Ten Tears in Chile

Angèle Martin, Paz Núñez-Regueiro et Carine Peltier

\section{OpenEdition}

Journals

Édition électronique

URL : http://journals.openedition.org/gradhiva/1962

DOI : 10.4000/gradhiva.1962

ISSN : $1760-849 x$

Éditeur

Musée du quai Branly Jacques Chirac

Édition imprimée

Date de publication : 24 novembre 2010

Pagination : 218-227

ISBN : 978-2-35744-029-6

ISSN : 0764-8928

Référence électronique

Angèle Martin, Paz Núñez-Regueiro et Carine Peltier, « Araucanie-Bruxelles-Paris : la collection Gustave Verniory au musée du quai Branly », Gradhiva [En ligne], 12 | 2010, mis en ligne le 24 novembre 2013, consulté le 02 mai 2019. URL : http://journals.openedition.org/gradhiva/1962 ; DOI : 10.4000/gradhiva.1962 


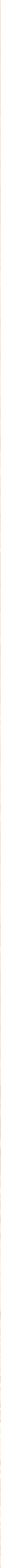

23. 


\section{Araucanie-Bruxelles-Paris : la collection Gustave Verniory au musée du quai Branly}

\section{Témoignages de dix années passées au Chili}

Angèle Martin

Paz Núñez-Regueiro Carine Peltier*
Fig. 1 Attribué à Gustavo Milet Ramirez (1860-1917), Indienne en grande toilette, vers 1885

(album 70.2010.11.51)

(c) musée du quai Branly.

\footnotetext{
- -

* Nos remerciements les plus sincères vont à Mylène, Jacques et Jean Massion, petits-enfants de Gustave Verniory, ainsi qu'à Jean Boxus pour leur généreuse donation et leur aide précieuse.
}

Parti au Chili en janvier 1889 pour participer à la construction du chemin de fer en Araucanie, au centre du pays, l'ingénieur belge Gustave Verniory (1865-1949) s'éprend de cette région où il a travaillé dix ans (1889-1899). Son récit de voyage et ses notes constituent une source historiographique de première importance sur la colonisation de l'Araucanie, qui suivit la défaite militaire des communautés mapuche, ou araucanes selon le terme en usage à l'époque. En juin 2009, le musée du quai Branly a acquis les tapuscrits de Verniory, partiellement inédits. Par la suite, les échanges fructueux établis avec ses descendants, toujours détenteurs des objets, photographies et écrits rassemblés au Chili, ont abouti en mars 2010 au don de cette collection conservée en Belgique depuis plus d'un siècle.

Un ingénieur au service de la «colonisation »

Sur ce territoire long de près de 5000 kilomètres qu'est le Chili, la construction des premières voies ferrées débute dans les années 1850 . Le chemin de fer s'étend dans le nord du pays sous la pression du secteur minier, d'un grand dynamisme exportateur. Il ne gagne les provinces du centre et du sud qu'après la "pacification de l'Araucanie", finalisée en 1883. Cette offensive armée conduite par le gouvernement chilien met fin à l'insurrection de plus de trois siècles des Mapuche dans les territoires au sud du Bío-Bío, fleuve qui matérialise pendant la plus grande partie du xix ${ }^{e}$ siècle une frontière, la "Frontera", au-delà de laquelle les communautés indigènes maintiennent leur indépendance et leur autonomie.

L'arrivée au pouvoir de José M. Balmaceda (1886-1891) marque un tournant dans la politique ferroviaire de l'État chilien, qui voit dans le chemin de fer le moyen d'étendre la frontière agricole et la colonisation vers le sud. Ce dernier doit également, selon les termes de Verniory, «assurer la défense des passages du sud 


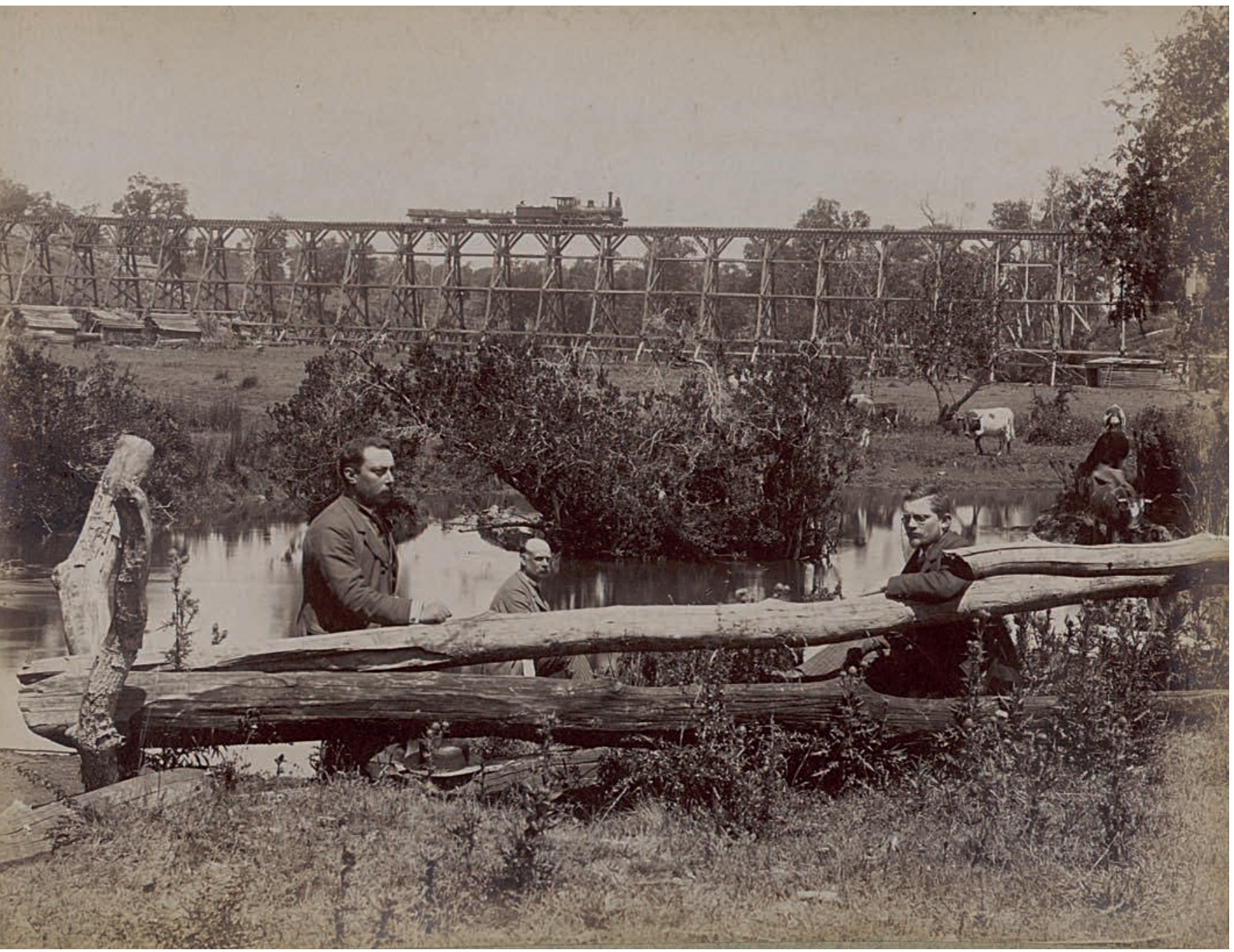

Fig. 2 Anonyme, Pont de Quillem, 1892 (album 70.2010.11.51). À droite : Gustave Verniory @ musée du quai Branly.

-

1. Dix Années en Araucanie, t. I : 56.

2. Alliende 1993, Guajardo Soto 200?.

3. La Belgique, grande puissance manufacturière depuis 1850 , sombre à la fin du du XIx ${ }^{\mathrm{e}}$ siècle dans une dépression économique qui conduit les ingénieurs belges, convoités pour la qualité de leur formation et leur expérience, à émigrer (Oris et Potelle 1995).

4. Son père étant décédé quelques mois avant son départ, il est chargé de famille. de la Cordillère en cas d'une guerre toujours possible avec l'Argentine ${ }^{1}$ » en raison de désaccords frontaliers entre les deux pays. À partir de 1888, le réseau est ainsi élargi aux régions des fleuves Bío-Bío, Cautín et Malleco, qui fournissent en blé, bétail, bois et charbon le marché national et la capitale Santiago².

C'est dans ce contexte que Verniory arrive au Chili en mars 1889: diplômé des universités de Liège et de Bruxelles, âgé de 24 ans, il est comme bon nombre de ses compatriotes ${ }^{3}$ sollicité par le gouvernement chilien pour participer à la construction de la ligne de chemin de fer devant unir le centre du Chili à la province isolée de Valdivia, accessible uniquement par la mer. Verniory part au Chili pour trois ans; il y restera une décennie. Ayant besoin de subvenir aux besoins de sa famille restée à Bruxelles4, il multiplie les missions et contrats sur place, pour des entreprises étrangères et nationales puis à son propre compte. Après une première impression négative de l'Araucanie, il développe une vraie passion pour la liberté que lui confère la vie à la Frontera. 


\section{Témoignages de voyage : récits, objets et images d'Araucanie}

Les quatre tapuscrits Dix Années en Araucanie (deux volumes), Scènes indiennes d'Araucanie et D'Araucanie en Europe par Panama, notes de voyage 1899 sont écrits par Verniory après 1935, durant ses premières années de retraite, et copiés en nombre restreint pour les membres de sa famille. Les deux volumes de Dix Années en Araucanie ont été publiés en espagnol par l'université du Chili en 1976 et $1981^{5}$, puis réédités par la maison d'édition Pehuén en 2001 et 2005. Les deux autres volumes sont totalement inédits. Tous prennent la forme d'un journal, excepté Scènes indiennes d'Araucanie, recueil de descriptions ethnographiques, matérielles et linguistiques sur les Mapuche.

La collection donnée au musée inclut un fonds d'archives principalement composé de correspondances datées de 1889 à 1904, qui offre un complément aux tapuscrits. La méticulosité de Verniory apparaît dans son classement, conservé par ses descendants : empaquetées par années, légendées de sa main, des liasses rangées chronologiquement distinguent les copies des lettres qu'il a envoyées à sa famille de celles qu'il a reçues. Ces échanges offrent un éclairage supplémentaire sur le quotidien et la personnalité de Verniory : nécessité financière du colon contraint à la réussite, solitude de l'exilé parti seul, excitation de la découverte d'un territoire et d'une culture. Certaines informations contenues dans les lettres n'apparaissent pas dans les tapuscrits, tels les débuts de la constitution de sa collection d'objets 6 .

Le fonds d'objets est composé de cinquante pièces représentatives de la culture mapuche de la fin du xix ${ }^{e}$ siècle : récipients en matériaux variés, plats à vanner, éléments de harnachement, éperons et étriers, bijouterie indigène en argent, blagues à tabac, bandes tissées trarilonko et trarihue, mais aussi un masque en bois, un kultrun ou tambour de chamane et un poteau funéraire chemamul. Ce dernier, pièce maîtresse de la collection, fut prélevé en avril 1896 dans un «eltun ou cimetière abandonné, à mi-chemin entre le Cautin et le Quepe ${ }^{7}$ ", dont plusieurs vues sont intégrées dans les albums photographiques. Le récit de Verniory nous renseigne ainsi souvent sur la façon dont il a obtenu les pièces (cadeau, troc, achat) et sur leur date d'envoi en Belgique.

Le corpus de photographies se compose de sept albums et d'une vingtaine de tirages volants, sur papier albuminé et aristotype, et d'un fascicule de reproductions imprimées, soit un ensemble de quelque deux cent cinquante images presque toutes légendées et le plus souvent datées de la main de Verniory. Principalement réalisés entre 1885 et 1899 par des photographes professionnels qu'il croise durant ses déplacements ou bien achetés en souvenir, les tirages sont rarement signés.

Trois albums se composent de photographies prises par des professionnels rendant compte à la fois des travaux de construction menés par Verniory et de la vie des Mapuche qu'il côtoie. Depuis le Chili, Verniory envoie à sa famille ces albums de souvenirs édités par Roberto Marks (actif 1877-1903). Un album moins officiel est consacré aux chantiers de construction. Deux autres, d'un intérêt plus ethnologique, sont entièrement consacrés à des photographies de Mapuche prises par les tout premiers professionnels établis en Araucanie. Enfin, un album et le fascicule imprimé par Félix Leblanc (actif 1865-1900) représentent des vues touristiques, les monuments des grandes villes (Santiago et Valparaiso) et des paysages que Verniory découvre durant son voyage du détroit de Magellan à Valparaiso en 1889.
- -

5. Le texte fut remis en 1963 par le petit-fils de l'auteur, Jean Massion, à Guy Santibanez, professeur de médecine.

6. Lettre de juin 1890, musée du quai Branly-Archives, inv. DA001206-66405.

7. Dix Années en Araucanie, t. II : 311. 
PAGE CI-CONTRE

Fig. 3 Épée en argent, blague à tabac et récipient céramique en forme de sirène, Araucanie, fin du xIx ${ }^{e}$ siècle, coll. musée du quai Branly ( $\mathrm{n}^{\circ}$ inv. 70.2010.11.14 70.2010.11.19 et 70.2010.11.50.1-2) (c) musée du quai Branly, photo Claude Germain.

\section{- $\bullet$ \\ 8. Dix Années en Araucanie, t. II : 294. \\ 9. Cerda Brintrup 2009.}

10 . $\ll$ Le roto chilien est $[. .$. le vrai descendant des anciens Indiens qui au cours des âges sont devenus la plèbe chilienne. » (Dix Années en Araucanie, t. I : 160)

11. Carlos Aldunate del Solar, communication personnelle, 2010.

12. Scènes indiennes, prologue : 1 .

13. Rudolf Lenz, «philologue allemand, professeur de langues à l'institut pédagogique de Santiago » (Dix Années en Araucanie, t. II : 310), est l'auteur de l'ouvrage Estudios araucanos: materiales para el estudio de la lengua, la literatura y las costumbres de los Indios mapuche o araucanos (Santiago, Imprenta Cervantes, 1895-1897).

14. Dix Années en Araucanie, t. II : 310.
L'Araucanie en construction : regards portés sur la Frontera

à la fin du xIXe siècle

Le processus d'occupation et d'urbanisation qui suit la pacification de l'Araucanie est rythmé par l'avancée du chemin de fer. Le récit de voyage et les albums photographiques documentent les étapes de ce chantier titanesque, telle la construction du pont sur le fleuve Malleco à Collipulli, encore inachevé lors de l'arrivée de Verniory en mars 1889. Chargé dans un premier temps de la mise en place des lignes de Victoria à Tolten, puis de Temuco à Pitrufquen, l'ingénieur belge inclut dans ses albums des vues des divers travaux menés par ses équipes : creusement des tranchées, construction des ponts de Perquenco, de Tricauco et de Ouillem (fig. 2) en 1892, puis ceux de Tolten et de Quepe en 1897, remblais, pose de rails, etc. Il fournit des vues de vastes territoires encore "sauvages » où s'installent les premières villes. À titre d'exemple, si en juillet 1895 Verniory écrit qu'«à un kilomètre de la rive on créera en plein bois la station de Quepe, amorce d'une nouvelle ville ${ }^{8}$ ", les diverses vues de l'inauguration de la station datées de 1896 attestent la concrétisation du projet urbain.

De 1883 à 1890, près de 10000 colons étrangers d'origine principalement allemande, française, anglaise, suisse et espagnole viennent s'établir dans la région ${ }^{9}$. Le récit de Verniory documente de manière inédite ce processus, sous ses aspects politique, économique, culturel et social. Les villes naissantes de Traiguén, Victoria, Lautaro, Pitrufquen, Pucón, Temuco, etc., sont le scénario de ces histoires, immortalisées dans ses albums: vues des bourgs naissants qui suivent la construction des voies du chemin de fer, tel le reportage photographique sur Nueva Imperial, et surtout portraits de la société dans laquelle il vit. Ces derniers documentent les différents «types " que Verniory côtoie : du Mapuche au «métis » et au Chilien en passant par le roto ${ }^{10}$, ses collègues étrangers et lui-même. Ils immortalisent un "Far West » à la chilienne qui n'existe déjà plus lorsqu'il quitte le pays en 1899 . Véritable tour de Babel, cette société se voit également condensée dans la collection d'objets de Verniory : une épée hispano-créole au manche en argent travaillé par un orfèvre mapuche, une blague à tabac décorée des fleurs de chilco locales par une tisserande indigène ou un récipient céramique en forme de sirène qui pourrait faire référence au mythe de la Pincoya de l'île voisine de Chiloé ${ }^{11}$ évoquent ce monde profondément métis (fig. 3).

Verniory a volontairement isolé ses observations les plus complètes dans Scènes indiennes d'Araucanie, volume à l'ambition clairement ethnographique dont le but est «de donner quelques renseignements complémentaires sur les mœurs des Araucans, d'autant plus que cette race intéressante est appelée à disparaître bientôt et que rares sont ceux qui se sont appliqués à conserver son souvenir ${ }^{12}$ ». Le texte s'organise en trois parties: après quelques informations sur l'alimentation, la production d'objets usuels et la langue mapuche, Verniory relate les "scènes indiennes " qu'il juge les plus marquantes parmi celles qu'il a eu l'occasion de vivre et d'observer, avant de proposer un petit recueil de contes indigènes. Ce travail semble avoir été en partie motivé par la collaboration engagée avec le Dr Rudolf Lenz en mars $1896^{13}$. Verniory s'emploie dès son arrivée à apprendre le mapuzungun, et sa relation privilégiée avec les caciques de la région lui vaut la visite du professeur lui demandant de mener des enquêtes sur la langue indigène auprès des Mapuche et « de chercher à reproduire les chants, les récits, les contes indigènes ${ }^{14} »$. 


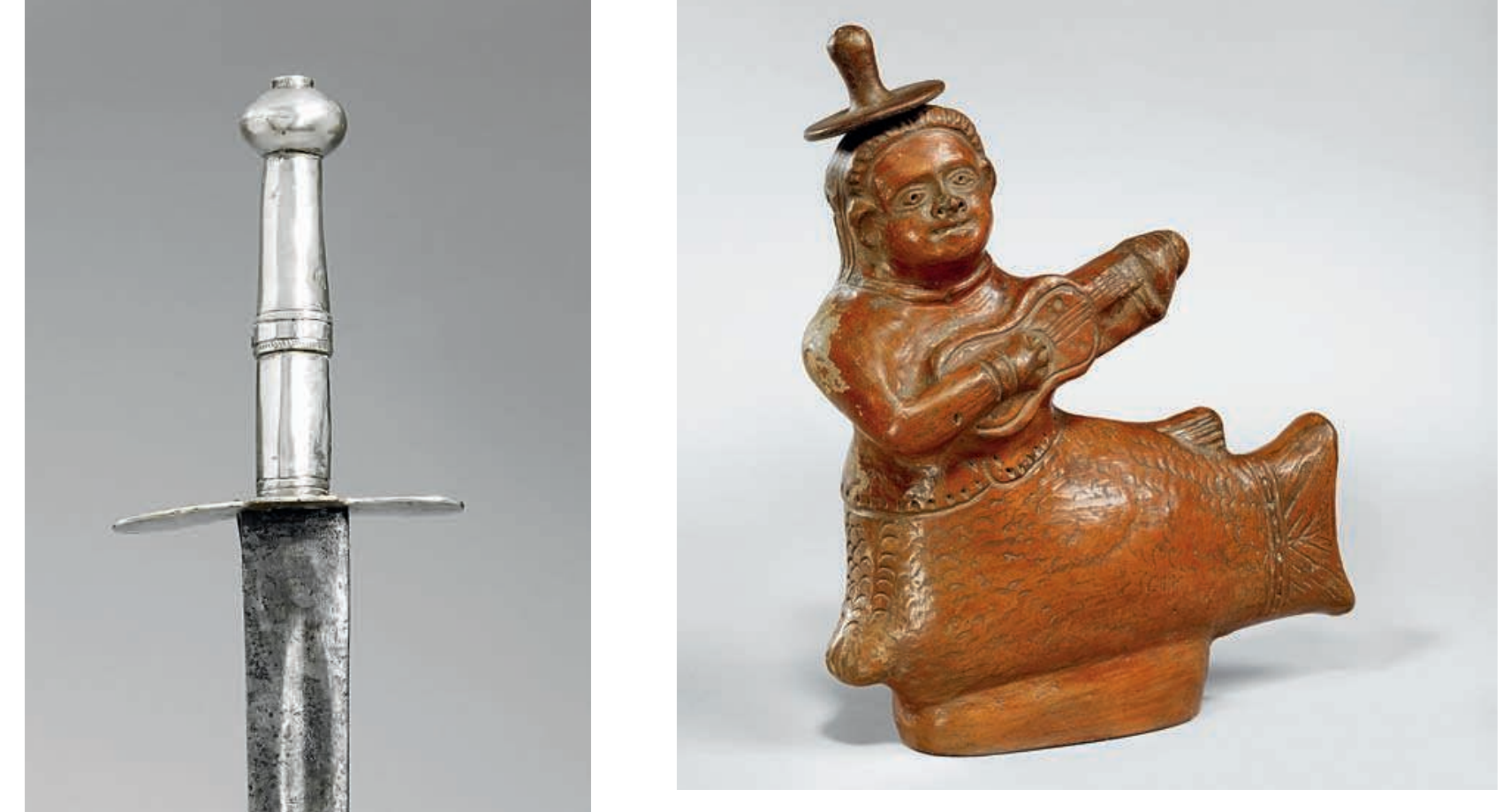




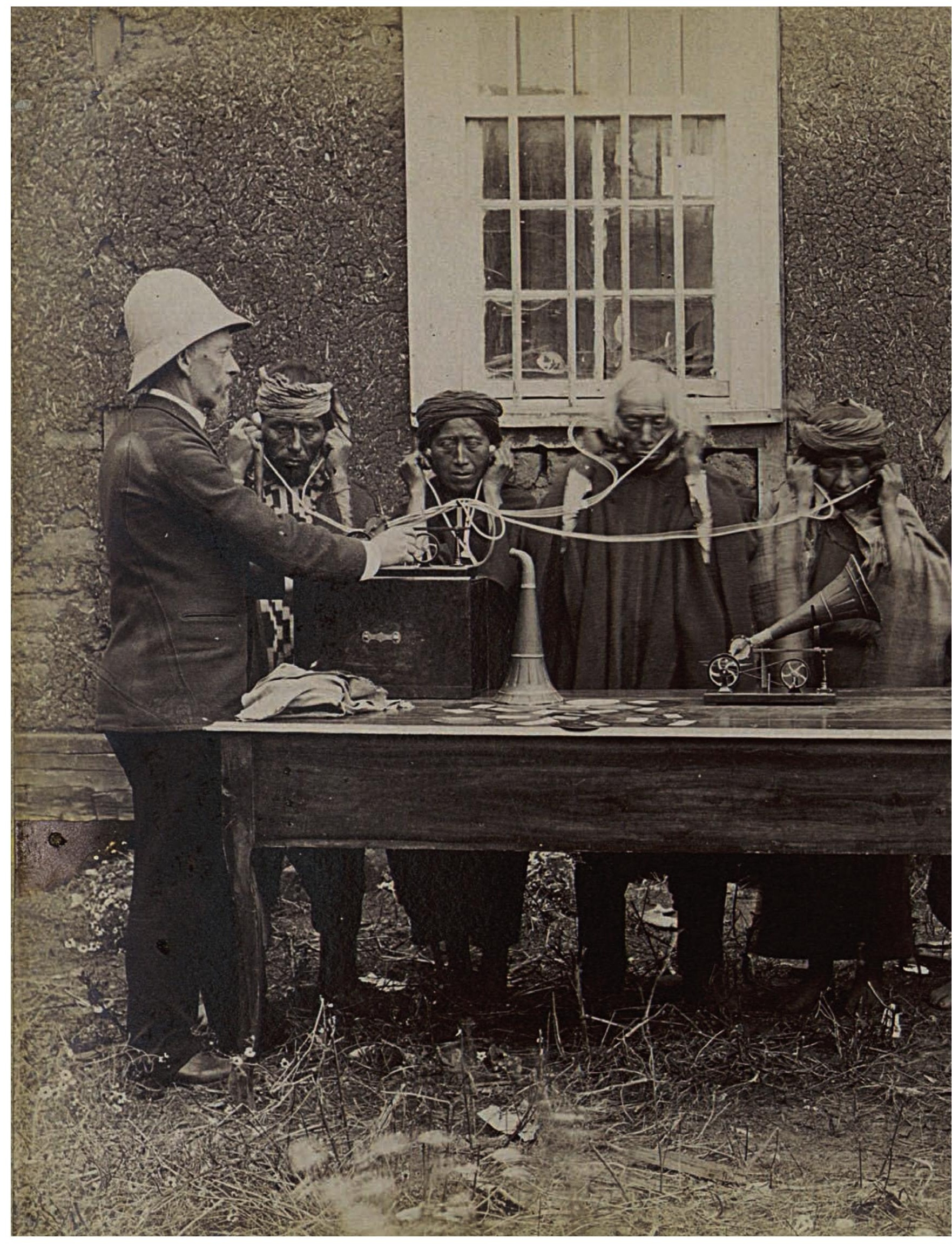




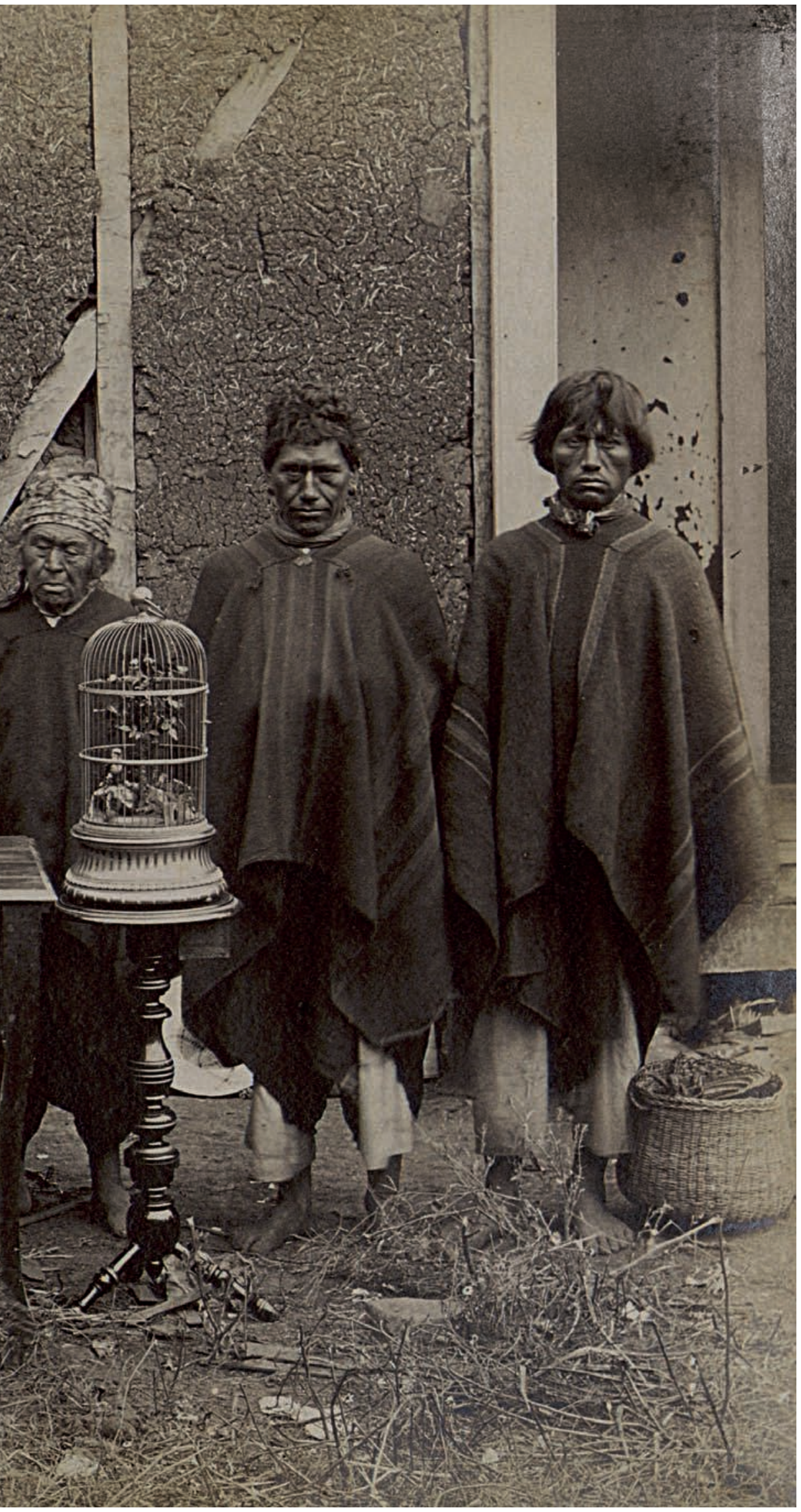

Fig. 4 Anonyme, Le Premier Phonographe en Araucanie, 1893 (album 70.2010.11.51)

(c) musée du quai Branly. 
-

15. Voir à ce sujet le travail d'Alvarado, Mege et Baez 2001.

16. Un seul est véritablement nommé : Victor Jottrand (18641912), explorateur et photographe belge de passage au Chili en novembre 1895

17. Dix Années en Araucanie, t. I : 194.

18. Mise en ligne déjà effective des objets de la collection sur le site Internet du musée ; installation dans les salles permanentes du poteau funéraire chemamul qui a fait l'objet d'une intervention de restauration-conservation; numérisation des albums photographiques pour leur mise en ligne et numérisation de la totalité des archives; publication intégrale en langue originale des tapuscrits de Verniory.
Ses observations sont complétées par les vues des albums photographiques, en particulier les deux volumes consacrés exclusivement à la culture mapuche, amplement annotés et contextualisés. Ceux-ci offrent un large panorama de la vie des Mapuche à la fin du xix siècle : la ruca (maison), les costumes traditionnels, le jeu du palin, le machi (chamane) avec son poteau cérémoniel rewe, les caciques et leurs familles, des portraits individuels ou de groupe et des cérémonies rarement photographiées comme la cérémonie religieuse du nguillatún et des scènes de funérailles.

Les recherches préliminaires sur le corpus de vues ethnologiques mettent en évidence qu'il est principalement l'œuvre de photographes professionnels, tels Obder Heffer Bissett (1860-1945), Gustavo Milet Ramirez (1860-1917) et B. Herrmann (actif 1885-1898?). Ces images, présentées comme documents fiables dans des ouvrages de sciences humaines et largement diffusées par la carte postale, répondent en réalité à une composition bien précise. Si cela semble plus évident pour les portraits pris en studio, comme celui de l'Indienne en grande toilette (fig. 1) où l'on devine le décor peint de l'arrière-plan, le parquet recouvert de paille et de bûches, et la pose rigide de la femme portant des vêtements et bijoux traditionnels (qui appartenaient en fait au photographe lui-même, voir Alvarado et al. 2001 : 23), on trouve également des mises en scène dans les prises de vues extérieures, en raison de contraintes techniques mais surtout pour répondre aux codes esthétiques et formels européens. Ces photographies ont largement participé à la construction d'un imaginaire collectif sur le peuple mapuche ${ }^{15}$.

Le travail mené sur le fonds photographique, en lien avec les sources écrites, devrait permettre d'éclairer les circonstances des prises de vues et de restituer l'identité de certains protagonistes mapuche, devenus archétypes visuels. Le récit et les notes sur les albums documentent certaines photographies reproduites dans divers ouvrages publiés dès la fin du xix siècle. Ces sources permettent d'identifier, sinon le nom des photographes ${ }^{16}$, du moins certains protagonistes indigènes restés dans l'anonymat jusqu'à aujourd'hui, et précisent des lieux et des dates de prises de vues. Ainsi, pour la très étonnante photographie qui met en scène des Mapuche écoutant «le premier phonographe en Araucanie » à Temuco (fig. 4), Verniory écrit en février 1893 : «Un photographe de passage a eu l'idée de s'entendre avec le Yankee [le propriétaire de l'appareil] pour prendre à l'écoute deux Indiens et deux Indiennes, photo qui se trouve dans un album à Bruxelles. À remarquer l'impassibilité absolue des traits des indigènes ${ }^{17}$. "

La collection Verniory apporte un éclairage inédit sur le processus de colonisation de l'Araucanie et sur la culture mapuche à la fin du xix siècle. Elle fait actuellement l'objet d'un projet de recherche mené conjointement par le service des Archives, l'unité patrimoniale des Amériques et l'iconothèque du musée du quai Branly, en association avec des collègues anthropologues et conservateurs chiliens. Dans les prochains mois, ce fonds précieux sera progressivement mis à disposition du public ${ }^{18}$. musée du quai Branly
ama@quaibranly.fr
pnr@quaibranly.fr
cpe@quaibranly.fr

mots clés / keywords: Chili // Chile • Araucanie // Araucania - Mapuche // Mapuche - musée du quai Branly // musée du quai Branly $\cdot$ photographie // photographs $\cdot$ récit de voyage // travel account. 


\section{Bibliographie}

\section{Aluiende E., María Piedad}

1993 Historia del Ferrocarril en Chile. Santiago, Pehuén Editores.

\section{Alvarado P., Margarita, Mege R.,} Pedro et BÁez A., Christián

2001 Mapuche. Fotografías siglos XIX y XX. Construcción y Montaje de un Imaginario. Santiago, Pehuén Editores.

\section{Cerda Brintrup, Gonzalo}

2009 «Arquitectura patrimonial de la región de La Araucanía, Chile », Arquiteturarevista 5(1) : 55-64.

\section{GuAJARDo Soto, Guillermo}

2007 Tecnología, Estado y Ferrocarriles en Chile, 1850-1950. Mexico, Fundación de los Ferrocarriles Españoles-Universidad Nacional Autónoma de México [«Colección de Historia Ferroviaria »].

Oris, Michel et Potelle, JeanFrançois

1995 «Les Wallons hors de la Wallonie », http://www.wallonie-en-ligne.net/1995_ Wallonie_Atouts-References/1995_ch18-1_Oris_ Michel.htm.

\author{
VERNIORY, Gustave \\ s.d. Dix Années en Araucanie, 1889-1899, \\ 2 tomes, tapuscrit. Musée du quai Branly- \\ Archives, inv. DA001191. \\ s.d. D'Araucanie en Europe par Panama, \\ notes de voyage 1899, tapuscrit. \\ Musée du quai Branly-Archives, \\ inv. DA001193. \\ s.d. Scènes indiennes d'Araucanie, \\ tapuscrit. Musée du quai Branly-Archives, \\ inv. DA001192.
}

\section{Résumé / Abstract}

Angèle Martin, Paz Núñez-Regueiro et Carine Peltier, AraucanieBruxelles-Paris : la collection Gustave Verniory au musée du quai Branly. Témoignages de dix années passées au Chili - L'ingénieur belge Gustave Verniory (1865-1949) se rend dans le centre et le sud du Chili pour participer à la construction du chemin de fer. II raconte ses dix années en Araucanie (1889-1899) dans un récit de voyage qui constitue la principale source historiographique sur l'histoire du Chili après la conquête de cette région en 1883. En mars 2010, ses héritiers ont fait don au musée du quai Branly de la collection d'objets, de photographies et d'archives rassemblée par leur aïeul pendant son séjour chilien.
Angèle Martin, Paz Núñez-Regueiro and Carine Peltier, AraucaniaBrussels-Paris: the Gustave Verniory Collection at the musée du quai Branly. Testament to his Ten Tears in Chile - In the late 19th Century, the Belgian engineer Gustave Verniory (1865-1949) travelled to the southern part of central Chile to help build a railway line. The ten years he spent in Araucania (1889-1899) are the subject of a travel account that constitutes the principal historiographic source for this region of Chile in the immediate postconquest period (from 1883 onwards). In March 2010, his descendants entrusted the collection of objects, photographs and archives he had gathered in Chile to the musée du quai Branly. 